\title{
Measurement of Power Radiation in Base Transceiver Station Using Quad Phone and Quadcopter
}

\author{
Prem Kumar $\mathrm{N}^{1}$, Raj Kumar $\mathrm{A}^{1}$, Sundra Anand ${ }^{1}$, E. N. Ganesh ${ }^{2}$ \\ and V. Prithiviraj ${ }^{3}$ \\ ${ }^{1}$ Final year B.E. (ECE), Rajalakshmi Institute of Technology, Tamil Nadu, India \\ ${ }^{2}$ Dean R\&I, Rajalakshmi Institute of Technology, Tamil Nadu, India \\ ${ }^{3}$ Professor, Dept. of Electronics and Communication Engg (ECE), \\ Rajalakshmi Institute of Technology, Tamil Nadu, India \\ Corresponding Authors: \{prem0504kumar; raj16993; sundra.ece; \\ profvpraj\}@gmail.com; dean.research@ritchennai.ediu.in
}

Received January 2015; Accepted March 2015;

Publication April 2016

\begin{abstract}
The communication protocols used in Base Transceiver Station (BTS) could be harmful to human species and other life forms in the ecosystem. The BTS used for these systems could emit radiations beyond safety threshold. Therefore, it is essential to monitor such power radiation levels from time to time. Manual readings at each BTS are strenuous and time consuming work. This paper proposes radiation measurement using Quad Phone assembled within the Quadcopter using android software. Quadcopter is a low cost restricted payload machine which suits the measurement of radiation emitted from antenna towers and power lines. An application is developed to monitor the power radiation emitted by each of the bands and the associated communication protocol by utilizing the Quad Phone. It can use CDMA, GSM, 3G and LTE protocols at the designated frequency bands. A Quad Phone incorporating the android application is used to record power radiation levels at several points around a single tower facilitated by the Quadcopter flight navigation system. The Wave Point navigation is a hardware module used to move around a target
\end{abstract}

Journal of Green Engineering, Vol. 5, 107-128.

doi: 10.13052/jge1904-4720.522

(c) 2016 River Publishers. All rights reserved. 
point up to 16 locations and circle back to the initial position. The collected data regarding the radiated signal strength for different protocols are either transmitted through wireless or stored within the Quad Phone which could be retrieved later. The power readings around each base station can be recorded using the Quad Phone and the Quadcopter in the shortest time possible. Hence the combination of the Quad Phone mounted on the Quadcopter provides an excellent monitoring system for auditing the Electromagnetic Radiation (ER) and subsequently determine the Electromagnetic Pollution Index (EPI) from the delineated pockets of pollution regions.

Keywords: Base Transceiver Station (BTS), Code Division Multiple Access (CDMA), Global system for Mobile Communications (GSM), Wave point navigation, Global Positioning System (GPS), Electromagnetic Radiation (ER).

\section{Introduction}

Quadcopter is a multirotor helicopter and is propelled by four rotors. In order to possess less Kinetic Energy during the flight period, each rotor which is mounted on the Quadcopter should have a smaller diameter when compared to the helicopter rotor. As the Quadcopter has its propeller pitch angle not being subjected to any variation, it results in a simpler design implementation for the copter. At present there are fewer vehicles capable of utilising vertical takeoffs and landing mechanisms [1]. The Base Transceiver Stations emits radiations which is being picked up by the cell phones and enables to establish a connection. Now-a-days in order to increase the cell coverage the service providers could radiate large amount of energy which could be harmful to the human species and other flora and fauna. To monitor over these radiation levels the Indian Ministry of Telecommunication formed a committee called TERM cells where the members of the committee move around each BTS measuring the signal strength for each service providers for every quarter. This method is found to be tedious as it requires large amount of human resource, vehicle field strength measurement and is a time consuming process.

This brought immediate attention and as a solution for this problem it is proposed to develop a frame work with a goal to develop an android application for monitoring the signal strength of the BTS at various locations utilizing a Quad Phone mounted within a Quadcopter. A quad phone is quad core quad band phone that works in multiple radio frequency bands using 
GSM/CDMA technology. The need for Quadphone is to measure the signal strength across four different cellular services like GSM, CDMA, HSDPA and LTE. Since Quad Phone is light weight smart phone it can be readily used with a Quadcopter to measure the signal strength and radiation level. The four rotors which are mounted in a cross combination provides the necessary lift force thereby enabling the Quadcopter to be airborne. A major distinguishing factor is due to the controlling mechanism of the Quadcopter which is different from the normal helicopter. Quadcopters possess a significant advantage compared to the fixed wing airplanes due to its maneuverability in all directions, with improved hovering capability and for flying at lower speeds.

The rotors mounted on the quadcopter are distinctly responsible for producing the desired thrust and torque around its center of rotation. A distinguishing point is that its propellers are not at all alike and this enables the quadcopter to operate under two pairs, one for establishing the pushing effect and the second pair for enabling the pulling effect, consequently enabling the resultant net torque to be null, provided all the propellers turn with the same angular velocity, thereby permitting the coptercraft to remain still around its center of gravity [7].

Figures 1 and 2 illustrates the movements of each rotor. Changes in the pitch angle are induced by contrary variation of speeds in propellers 1 and 3

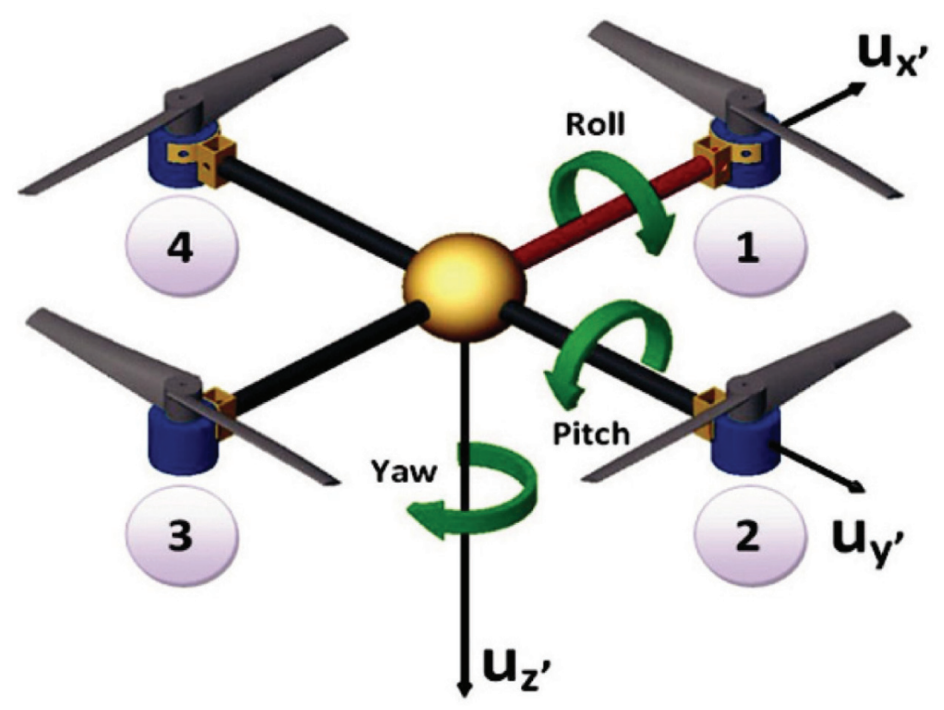

Figure 1 Yaw, pitch and roll rotations of a Quadcopter [7]. 



Low speed

High speed

Figure 2 Various movements of a Quadcopter [2].

(see Figure 1), resulting in forward or backwards translation. If we do this same action for propellers 2 and 4, we can produce a change in the roll angle and we will get lateral translation. Yaw is induced by mismatching the balance in aerodynamic torques (i.e. by offsetting the cumulative thrust between the counter-rotating blade pairs). So, by changing these three angles in a Quadcopter we are able to make it maneuver in any direction.

\section{Quadcopter Design}

The most important target of this particular design process is to arrive at the correct set of requirements for the copter with appropriate payload which 
could be summarized into a set of specifcations. The specifcations for the Quadcopter prototype are given below:

- Flight autonomy between 15 to 25 minutes.

- On-board controller should have its separate power supply to prevent simultaneous engine and processor failure in case of battery loss.

- Ability to transmit live telemetry data and receive movement orders from a ground station wirelessly, therefore avoiding the use of cables which could become entangled in the aircraft and cause an accident;

- Quadcopter should not fly very far from ground station so there is no need of long range telemetry hardware and the extra power requirements associated with long range transmissions.

Consequently, the main components are:

- 4 electric motors and 4 respective Electronic Speed Controllers (ESC).

- 4 propellers.

- 1 on-board processing unit with inbuilt Accelerometer, Gyroscope, Barometer and Magnetometer.

- 2 on-board power supplies (batteries), one for the motors and another to the processing unit. At this point we will assume that beyond the need to provide electrical power to the motors, we must assure that the brain of the Quadcopter (i.e. the on-board processing unit) remains working well after the battery of the motors has discharged;

- 1 airframe for supporting all the aircraft's components.

\subsection{Airframe}

The airframe is the mechanical structure of an aircraft that supports all the components, much like a "skeleton" in Human Beings. Designing an airframe from scratch involves important concepts of physics, aerodynamics, materials engineering and manufacturing techniques to achieve certain performance, reliability and cost criteria. We have designed the airframe of our Quadcopter with $258 \mathrm{~g}$ of mass and made of GPR (Glass-Reinforced-Plastic), possessing a cage-like structure in its center that will offer extra protection to the electronics.

This particular detail may prove itself very useful when it comes to the test flight stage, when accidents are more likely to happen. 
112 N. Prem Kumar et al.

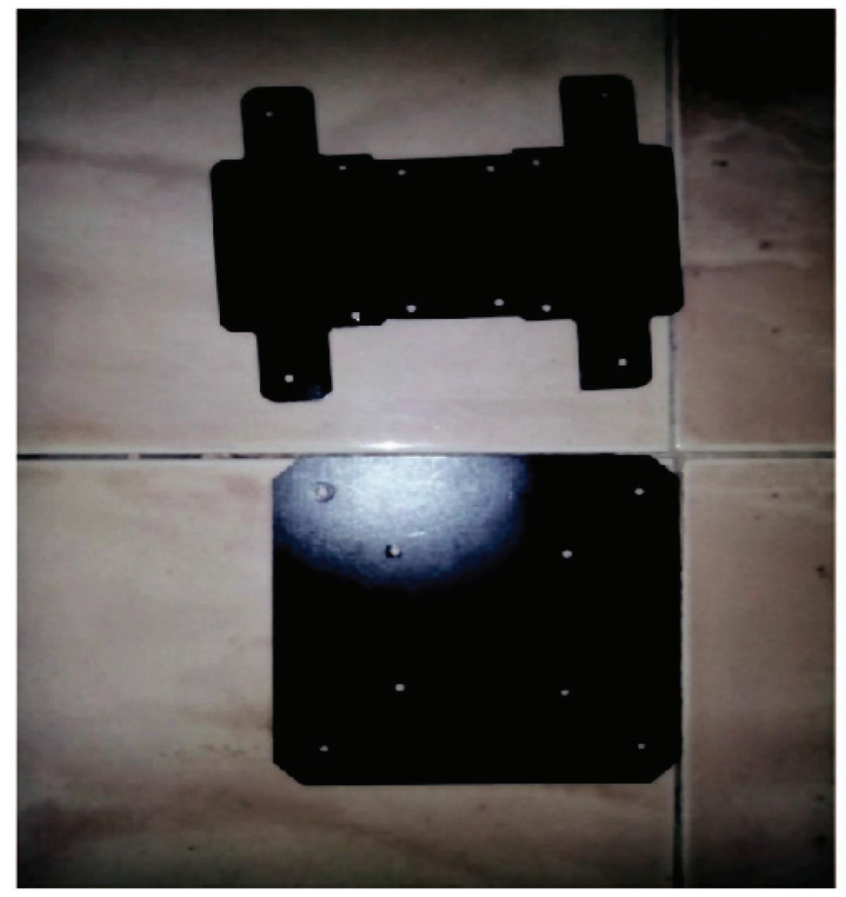

Figure 3 Top and the bottom plate.

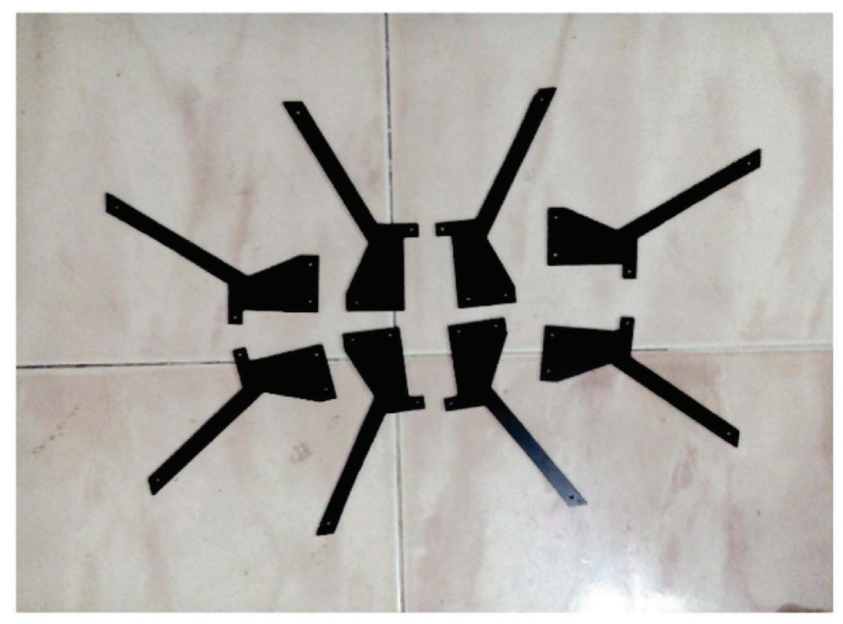

Figure 4 Quadcopter legs. 


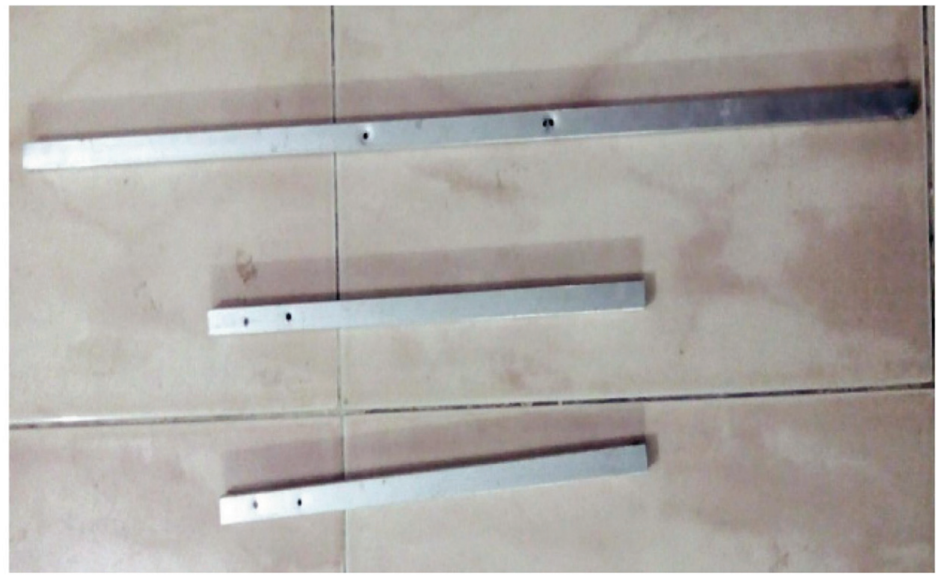

Figure 5 Aluminum frame.



Figure 6 Quad-X frame.

\subsection{Propellers}

The typical behavior of a propeller can be defined by three parameters:

- Thrust coefficient $\mathrm{c}_{\mathrm{T}}$;

- Power coefficient $c_{P}$;

- Propeller radius $r$. 


\section{N. Prem Kumar et al.}

These parameters allow the calculation of a propeller's thrust T:

$$
\mathrm{T}=\frac{\mathrm{c}_{\mathrm{T}} 4 \rho \mathrm{r}^{4} \omega^{2}}{\pi^{2}}
$$

and power

$$
\mathrm{P}_{\mathrm{P}}:=\frac{\mathrm{c}_{\mathrm{P}} 4 \rho \mathrm{r}^{5} \omega^{3}}{\pi^{3}}
$$

where $\omega$ is the propeller angular speed and $\rho$ the density of air. These formulas show that both thrust and power increase greatly with propeller's diameter. If the diameter is big enough, then it should be possible to get sufficient thrust while demanding low rotational speed of the propeller. Consequently, the motor driving the propeller will have lower power consumption, giving the Quadcopter higher flight autonomy. Available models of counter rotating propellers are scarce in the market of radio controlled aircrafts.

The "EPP1045" (see Figure 7), a propeller with a diameter of 10" $(25.4 \mathrm{~cm})$ and weighting $23 \mathrm{~g}$, presented itself as a possible candidate for implementation in the Quadcopter. To check its compatibility with the project requisites it is necessary to calculate the respective thrust and power coefficient. We can extract the mean thrust and power co-efficient by using Equations (1) and (2):

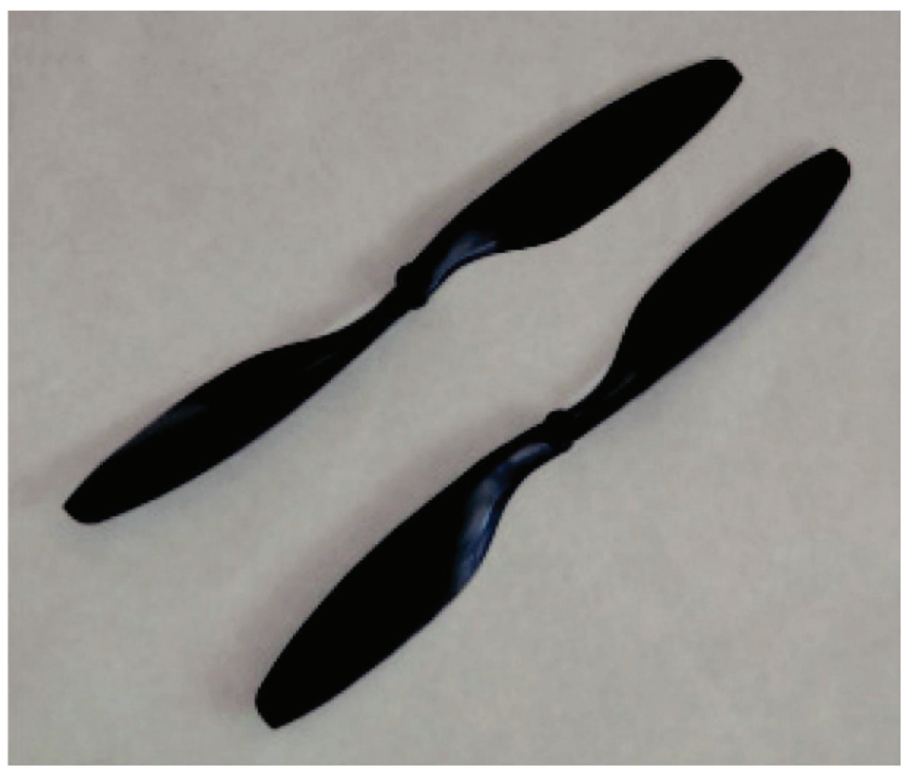

Figure 7 EPP1045 propellers. 


$$
\begin{aligned}
& \mathrm{c}_{\mathrm{T}}=0.1154 \\
& \mathrm{c}_{\mathrm{P}}=0.0743
\end{aligned}
$$

In reality, neither the thrust nor the power co-efficient are constant values, they are both functions of the advance ratio J:

$$
\mathrm{J}=\mathrm{u}_{0} \mathrm{nD}_{\mathrm{P}}
$$

where $u_{0}$ is the aircraft flight velocity, $n$ the propeller's speed in revolutions per second and finally $\mathrm{D}_{\mathrm{P}}$ is the propeller diameter.

However, when observing the characteristic curves for both these coefficient as shown in Figures 8 and 9, it is clear that when an aircraft's flight velocity is very low (e.g. in a constant altitude hover) the advance ratio is almost zero and the two co-efficient can be approximated as constants, which is the current case, for at this point there is no interest in achieving high translation velocity. Assuming the Quadcopter's maximum weight is $9.81 \mathrm{~N}$ $(1 \mathrm{~kg})$ and that we have four propellers, it is mandatory that each propeller is able to provide at least $2.45 \mathrm{~N}$ (1/4 of the Quadcopter weight) in order to achieve lift-off. Taking this data into consideration leads us to wonder about the minimum propeller rotational speed involved, as well as the magnitude of the power required for flight. Figure 10 helps us with some of these questions.

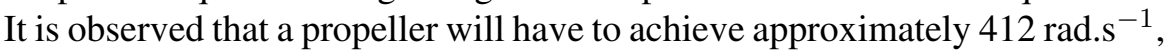

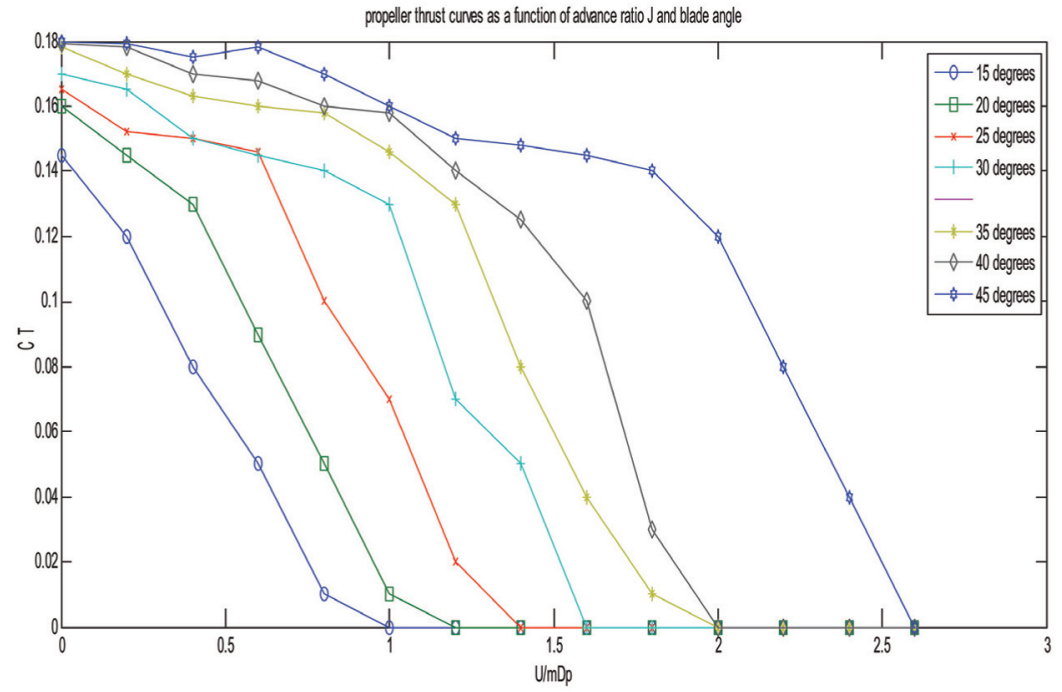

Figure 8 Typical propeller thrust curves as a function of advance ratio $\mathrm{J}$ and blade angle [8]. 


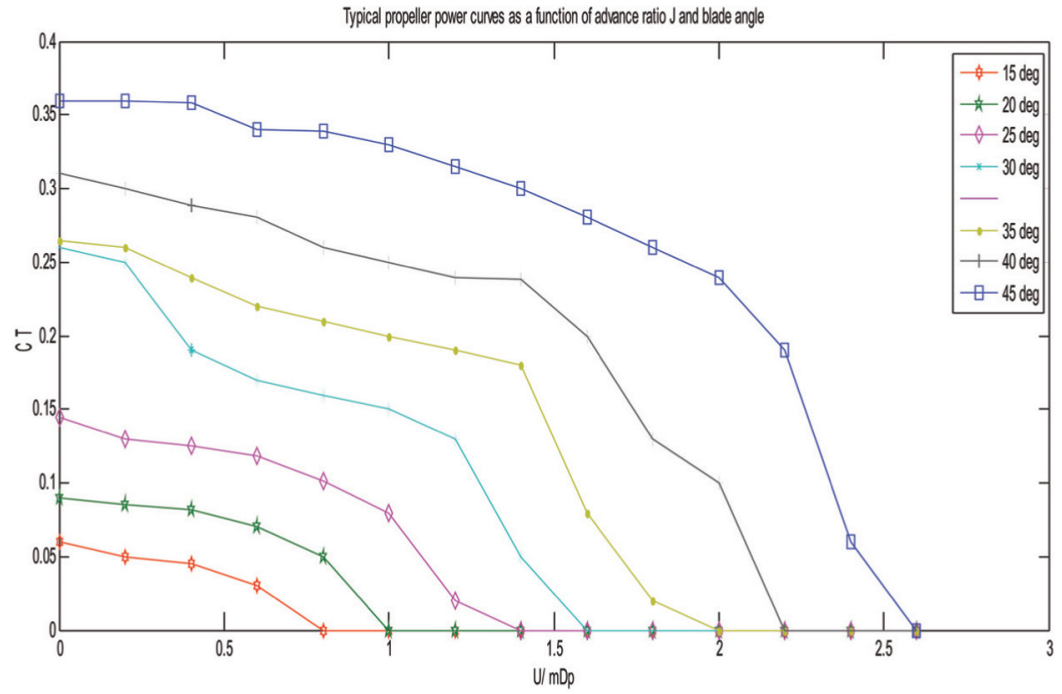

Figure 9 Typical propeller power curves as a function of advance ratio $\mathrm{J}$ and blade angle [8].

which is equivalent to 3934 revolutions per minute, to provide the minimum $2.45 \mathrm{~N}$ required for lift-off [6].

The respective propeller power is $26 \mathrm{~W}$. After this short analysis we can state that the EPP1045 propellers are suitable for implementation in the Quadcopter prototype.

\section{Multi-Wii Configuration}

The Multi Wii Copter is an open source software which controls the RC Platform and also compatible with the hardware boards and sensors.

The first and most famous setup is the association of a Wii Motion Plus and a Arduino pro mini board. The MultiWii 328P is a gyro/accelerometer based flight controller that is loaded with features. This version of the MultiWii supports DSM2 satellite receiver functionality. With expandability options and full programmability, this device can control just about any type of aircraft. This is the ideal flight controller for your multi-rotor aircraft. The pin diagram for MultiWii 328P is given in Figure 11.

\subsection{A Gimbals}

A gimbals is a pivoted support that allows the rotation of an object about a single axis. A set of three gimbals, one mounted on the other with orthogonal 

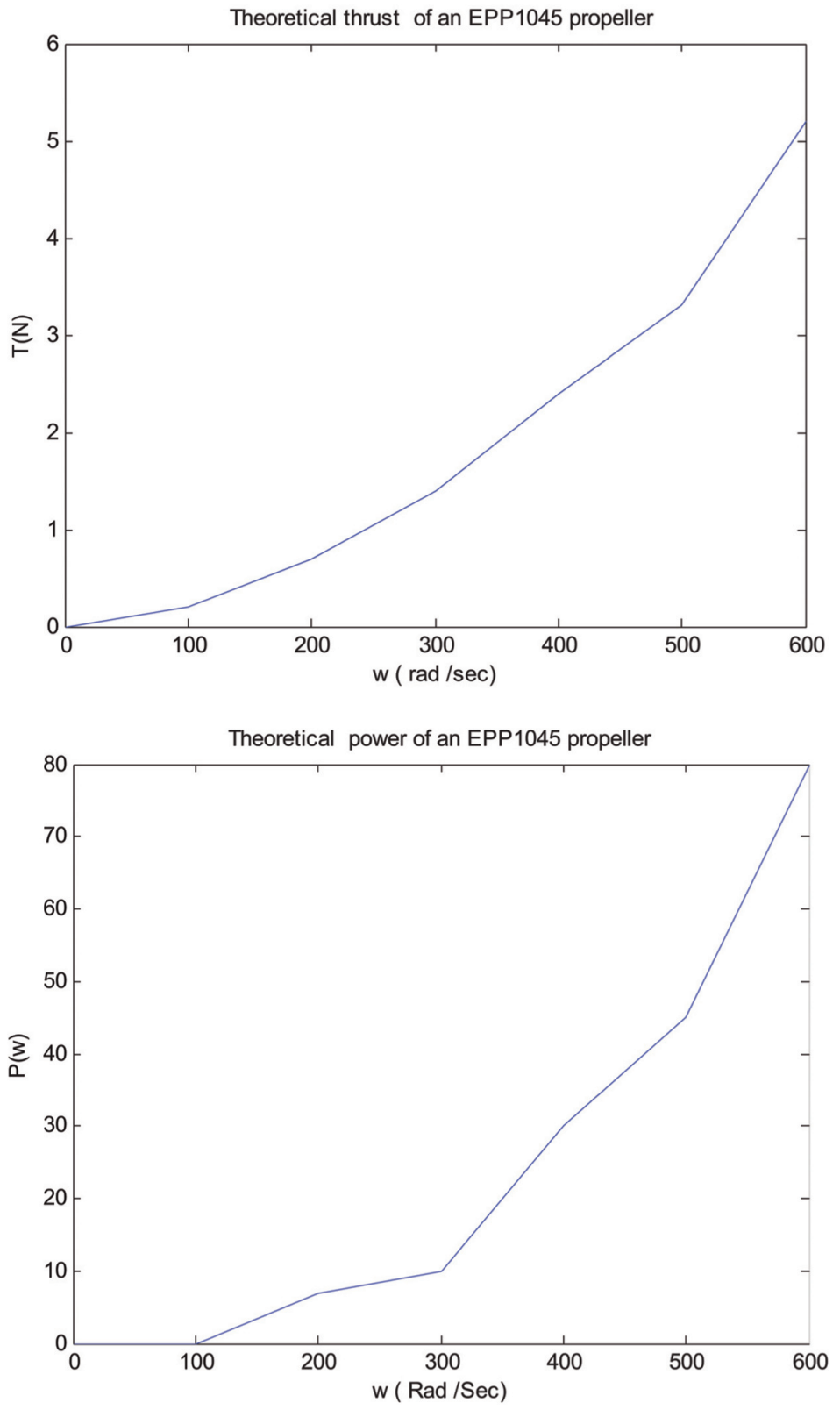

Figure 10 Theoretical thrust and power of an EPP1045 propeller. 


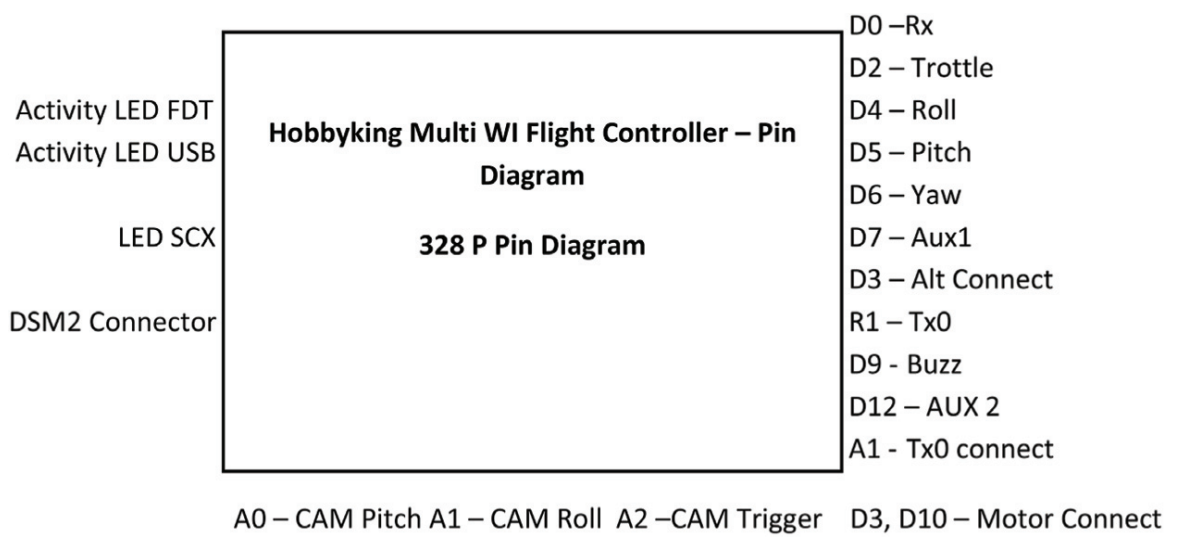

Figure 11 Pin diagram of MultiWii 328P.

pivot axes, may be used to allow an object mounted on the innermost gimbal to remain independent of the rotation of its support. For example, on a ship, the gyroscopes, shipboard compasses, stoves, and even drink holders typically use gimbals to keep them upright with respect to the horizon despite the ship's pitching and rolling. When associated with an accelerometer, MultiWii is able to drive 2 servos for PITCH and ROLL gimbal system adjustment. The gimbal can also be adjusted via 2 RC channels.

\subsection{B ITG3205 Triple Axis Gyro [9]}

This is a breakout board for InvenSense's ITG-3205, a groundbreaking triple-axis, digital output gyroscope. The ITG-3205 features three 16-bit analog-to-digital converters (ADCs) for digitizing the gyro outputs, a userselectable internal low-pass filter bandwidth, and a Fast-Mode I2C (400kHz) interface. Additional features include an embedded temperature sensor and a $2 \%$ accurate internal oscillator. The ITG-3205 can be powered at anywhere between 2.1 and 3.6 V. For power supply flexibility, the ITG-3205 has a separate VLOGIC reference pin (labeled VIO), in addition to its analog supply pin (VDD) which sets the logic levels of its serial interface. The VLOGIC voltage may be anywhere from $1.71 \mathrm{~V}$ min to VDD max. For general use, VLOGIC can be tied to VCC. The normal operating current of the sensor is just $6.5 \mathrm{~mA}$.

Communication with the ITG-3205 is achieved over a two-wire (I2C) interface. The sensor also features a interrupt output, and an optional clock input. 
A jumper on the top of the board allows you to easily select the I2C address, by pulling the $\mathrm{AD}_{0}$ pin to either VCC or GND; If CLKIN pin is not used jumper shoul be shorted on the bottom of the board to tie it to GND.

\subsection{BMA180 Accelerometer [9]}

This is a breakout board for Bosch's BMA180 three-axis, ultra-high performance digital accelerometer. The BMA180 provides a digital 14-bit output signal via a 4-wire SPI or I2C interface. The full-scale measurement range can be set to $\pm 1 \mathrm{~g}, 1.5 \mathrm{~g}, 2 \mathrm{~g}, 3 \mathrm{~g}, 4 \mathrm{~g}, 8 \mathrm{~g}$ or $16 \mathrm{~g}$. Other features include programmable wake-up, low-g and high-g detection, tap sensing, slope detection, and selftest capability. The sensor also has two operating modes: low-noise and low-power.

This breadboard friendly board breaks out every pin of the BMA180 to an 8-pin, 0.1 " pitch header. The board doesn't have any on-board regulation, so the provided voltage should be between 1.62 and $3.6 \mathrm{~V}$ for VDD and 1.2 to $3.6 \mathrm{~V}$ for VDDIO. The sensor will typically only consume $650 \mathrm{uA}$ in standard mode.

\subsection{BMP085 Barometer [9]}

This precision sensor from Bosch is the best low-cost sensing solution for measuring barometric pressure and temperature. Because pressure changes with altitude you can also use it as an altimeter! The sensor is soldered onto a PCB with a $3.3 \mathrm{~V}$ regulator, I2C level shifter and pull-up resistors on the I2C pins.

\subsection{E HMC5883L Magnetometer [9]}

The Honeywell HMC5883L is a surface-mount, multi-chip module designed for low-field magnetic sensing with a digital interface for applications such as low-cost compassing and magnetometer. The HMC5883L includes our state-of-the-art, high-resolution HMC118X series magneto-resistive sensors plus an ASIC containing amplification, automatic degaussing strap drivers, offset cancellation, and a 12-bit ADC that enables $1^{\circ}$ to $2^{\circ}$ compass heading accuracy. The I2C serial bus allows for easy interface. The HMC5883L is a $3.0 \times 3.0 \times 0.9 \mathrm{~mm}$ surface mount 16-pin leadless chip carrier (LCC). Applications for the HMC5883L include Mobile Phones, Netbooks, Consumer Electronics, Auto Navigation Systems, and Personal Navigation Devices. 
The HMC5883L utilizes Honeywell's Anisotropic Magneto Resistive (AMR) technology that provides advantages over other magnetic sensor technologies. These anisotropic, directional sensors feature precision in-axis sensitivity and linearity.

\section{Graphical User Interface}

Java language is used to code in Linux platform. GUI is developed for graphical visualization of sensors, processing control of motors in Quadcopter utilizing RC Signalling.

Figure 12 shows the Multi Wii Simulation for Quadcopter with GUI. It gives flying path, speed, latitude and longitude.

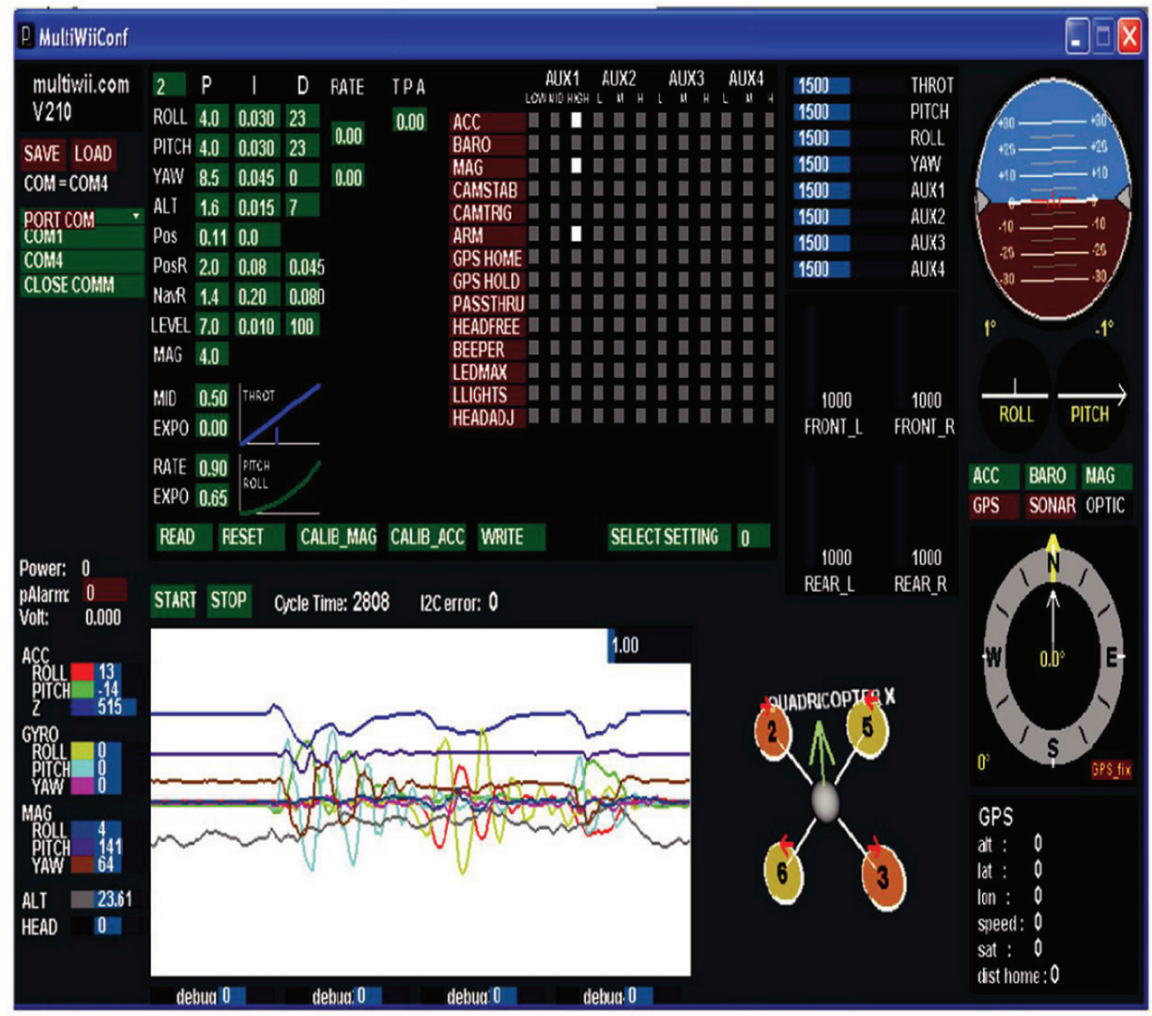

Figure 12 MultiWii Simulation for Quadcopter. 


\section{Android Application (SSM)}

Android application is developed for measuring the signal strength through Quad Phone. Android app created in the phone has user interface with the following parameters are created.

- IMEI Number

- Cell ID

- Signal Strength in $\mathrm{dBm}$

- EVDO Value

- SNR Value

- Button to fetch Details

Once this button is clicked all the details are displayed on the screen. Android app requires minimum of $1 \mathrm{Mb}$ memory requirement. All these contents are visible in the User Interface of the SSM application on the quad phone, which is placed over the Quadcopter as shown in Figures 13 and 14.

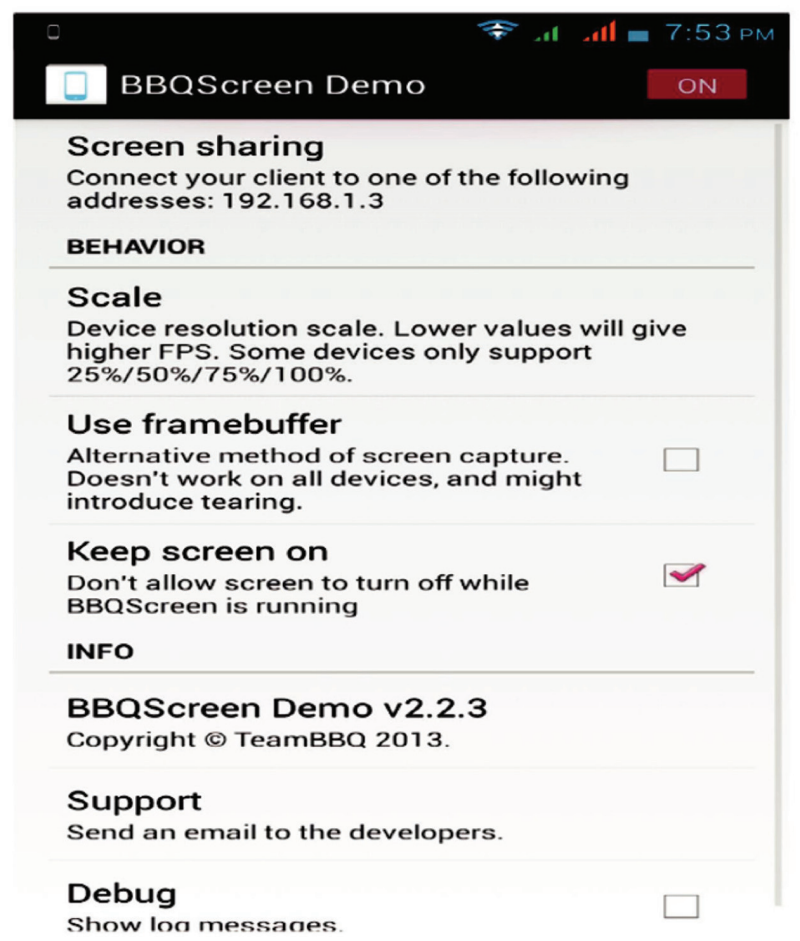

Figure 13 Mobile version of the BBQ application. 


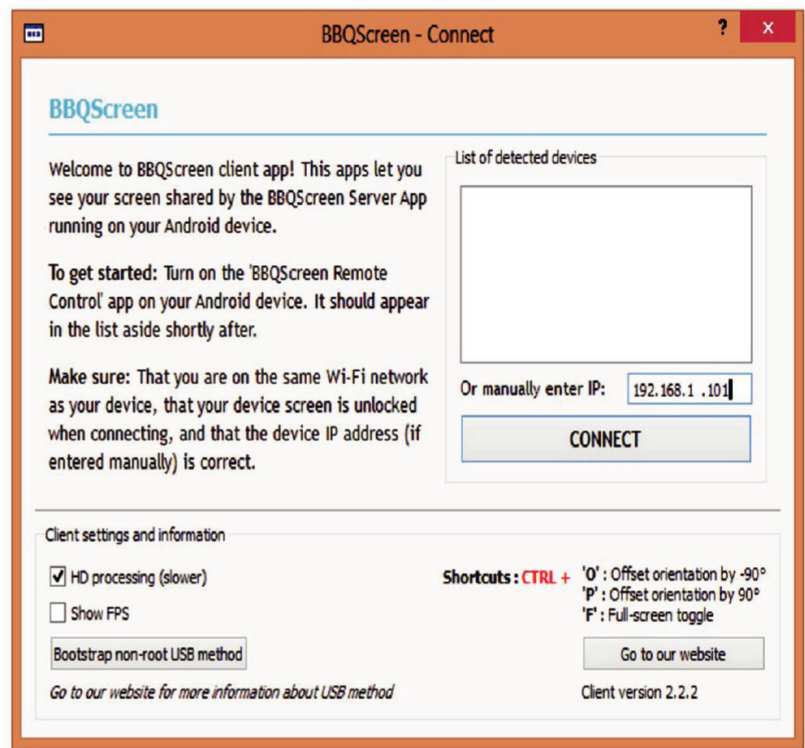

Figure 14 PC version of the BBQ client to view the phone screen on PC.

In order to view the signal strength displayed on the screen of the quad phone in PC, a new application BBQ is used. This BBQ requires the client software to be installed in the PC which is used to view the screen of the mobile phone [3]. BBQ is installed with the demo shown in Figure 13. Now the Quad Phone on the Quadcopter and the PC at the service end are connected to Wi-fi.

When BBQ is started it connects to IP automatically and this IP is connected to Mobile phone. Prior to this IP is given in BBQ software as shown in Figure 14 and thus a wireless connection between the PC and the quad phone is created. Using this wireless connection the screen of the quad phone can be viewed on the monitor of PC. Figure 15 shows the final picture of Quadcopter with Quad Phone. Figures 16(a) and 16(b) are screen shot of $\mathrm{BBQ}$ for measuring the signal strength.

\section{Results and Conclusion}

In this project, the development of the hardware and software framework necessary is undertaken to enable the Quadcopter to fly autonomously. The associated mechanical and electrical hardware is assembled and tested for 


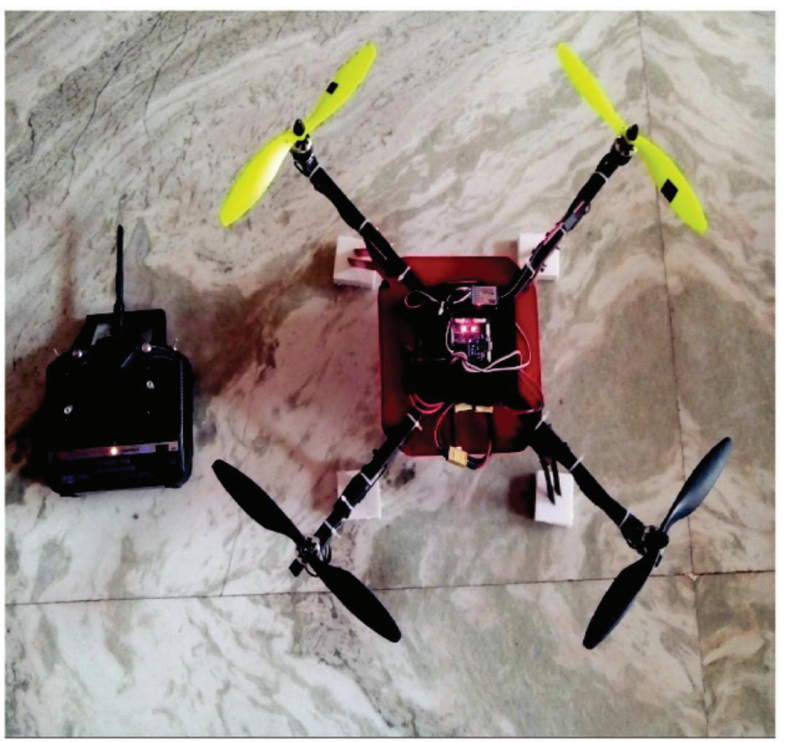

Figure 15 Final picture of the proposed Quadcopter.

its viability. The efficient design of Quadcopter housing the Quad Phone is utilized to measure the Signal Strength, SNR and and also to display the IMEI, cell ID and EVDO values associated with the Quad Phone. On analysis it is found that the proposed method of Quadcopter design developed provides a document that clearly and precisely outlines the steps necessary to assemble and fly the Quadcopter. With the implemented control scheme, the Quadcopter is able to hover autonomously and perform step movements in all directions. The experiments for several testing flying session have been performed for tuning the weight matrices of the controller and to carry out performance tests.

The main objective of this paper is to measure the radiated signal strength from a single Base Transceiver Station. This is achieved by mounting Quad Phone on a Quadcopter. An android application named "Signal Strength Monitoring (SSM)" is developed to monitor the power radiations emitted by each band i.e CDMA, GSM, HSPA, LTE in the communication protocol. So the mobile phone incorporating such an application is mounted on a Quadcopter to record the power radiation levels at several points around a single tower. Two signal strength values of $14 \mathrm{dbm}$ and $11 \mathrm{dbm}$ is noted for two different field tests carried out with SNR of -1 and EVDO value of -120 . The Signal 
124 N. Prem Kumar et al.

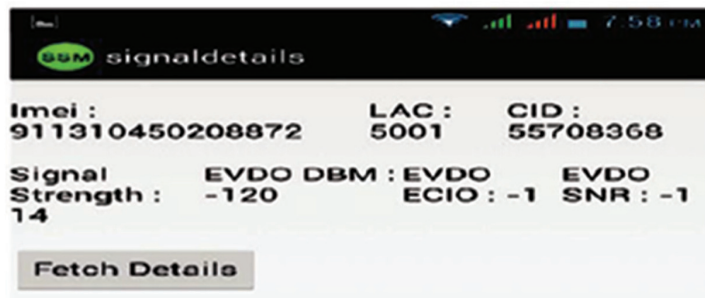

(a)

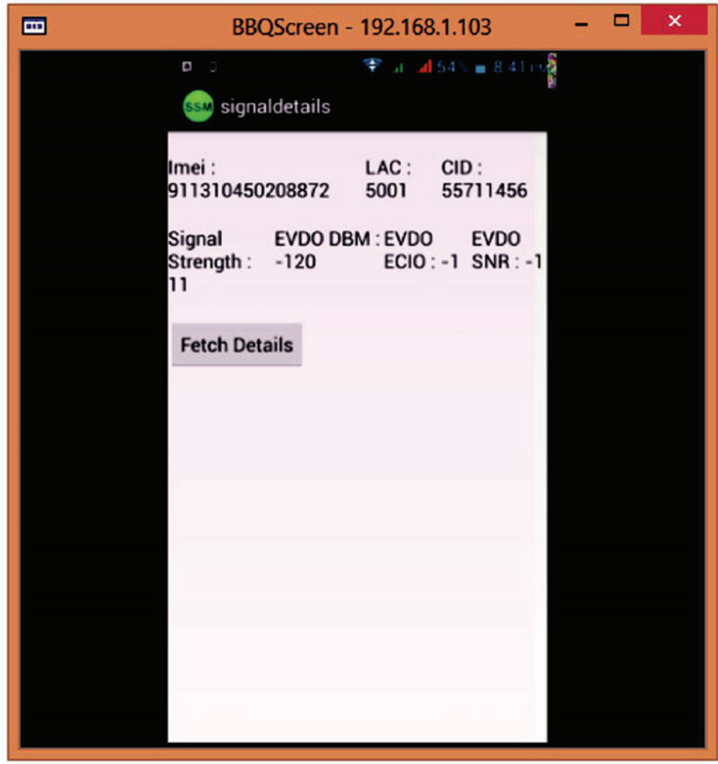

(b)

Figure 16 (a) Screenshots of the SSM Application over cell phone, (b) Screenshot of the application shared over the laptop.

Strength that is displayed by the Android Application (SSM) is successfully shared over a laptop using BBQ Software using the Wi-Fi connection and IP address. 
The above design encompassing a Quad Phone within the Quadcopter could pave way for undertaking Electromagnetic Pollution Index surveys within sensitive zones, City Malls, Railway Stations, Hospital zones and Airport restricted areas etc., [5]. This technique would carve out a distinct possibility for monitoring the Electromagnetic radiation in a residential complex like multi storied buildings/flats which are directly in the line of sight of the radiating tower antennas catering to various service providers. The combination of the Quadcopter and the Quad Phone would enable auditing of the Electromagnetic Radiation (ER) and subsequently determine the Electromagnetic Pollution Index (EPI) from the delineated pockets of pollution regions.

\section{References}

[1] Mary, C. L. C. Totu, L. C., and Koldbæk, S. (2010). Modelling and Control of Autonomous Quad-Rotor. Aalborg: Aalborg University.

[2] Jeremia, S., Kuantanna, E., and Pangaribuan, J. (2012). "Design and construction of remote-controlled quad-copter based on STC12C-5624AD," in Proceeding of the System Engineering and Technology (ICSET), 2012 International Conference, 1-6.

[3] Malathi, S., TirumalaRao, G., Rajeswer Rao, G., (2013). A prediction model for electromagnetic pollution index of multi system base stations. Int. J. Eng. Res. Technol. 2, 12.

[4] Mattar, N. A. B., Razak, M. R. B. A., Murat, Z. B. H., Khadri, N. B., and Rani, H. N. B. H. M. (2002). Measuring and analyzing the signal strength for Celcom GSM [019] and Maxis [012] in UiTM Shah Alam campus. Res. Dev. 2002, 489-493.

[5] Prithiviraj, V., Cmde, J. J. N., Avadhanulu, J. (2012). "Electromagnetic pollution index- a keyattribute of green mobile communications," in Proceeding of the Green Technologies Conference, 2012 IEEE, Washington, DC, $1-4$.

[6] Zhang, Y., Tianjin, K., Xian, B., Yin, Q., and Liu, Y. (2012). "Autonomous control system for the quadrotor unmanned aerial vehicle," in Proceeding of the Control Conference (CCC), Nanjing, 4862-4867.

[7] Jorge, M., and Brito, D. (2009). "Quadrotor prototype", in Instituto Superior Técnico, Portugal.

[8] Prof. Z. S. Spakovszky, Unified: Thermodynamics and Propulsion. Available at: https://http://web.mit.edu/16.unified/www/FALL/thermodynami cs/notes/node86.html

[9] Available at: https://http://www.geeetech.com/wiki 


\section{Biographies}



N. P. Kumar obtained his Bachelor of Engineering in Electronics and Communication Engineering from Rajalakshmi Institute of Technology, Chennai in 2014. Currently he is working as Backup Administrator. His areas of interests are Electronics \& Circuits, Digital Circuitry and Robotics.

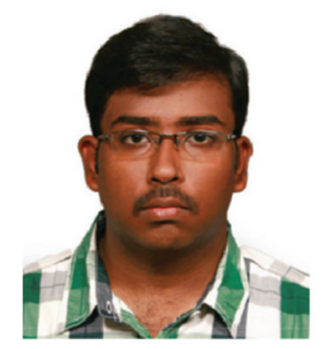

A. R. Kumar has completed his Bachelor of Engineering (Electronics and Communication Engineering) in 2014 from Rajalakshmi Institute of Technology, Chennai. Currently he is working as Assistant System Engineer in Tata Consultancy Services. His areas of interest include Robotics, Digital Electronics and Mobile Communication.

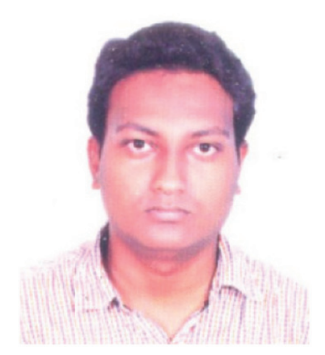

S. Anand obtained his Bachelor of Engineering in Electronics and Communication Engineering from Rajalakshmi Institute of Technology, Chennai in 
2014. Currently he is working as Engineer-Trainee. His areas of interests include Networking and Digital Circuitry.

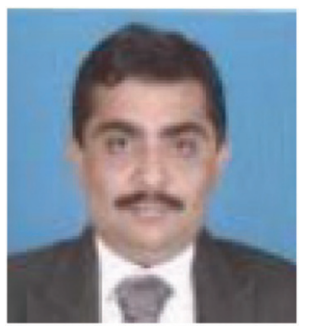

E. N. Ganesh received M.Tech. degree in Electrical Engineering from IIT Madras, Ph.D. from JNTU Hyderbad. He has over 20 years of academic experience and now working as Dean (Research and Innovation) at Rajalakshmi Institute of Technology. His area of interests is Nanoelectronics, Robotics and Hyperspectral Image Processing.



V. Prithiviraj received M.S. degree in Electrical Engineering from IIT Madras., Ph.D. in Electronics and Electrical Communication Engineering from IIT Kharagpur. He is working as Principal Rajalakshmi Institute of Technology from May 2013. He has over 3 decades of teaching experience and 12 years of Research \& Development Experience between the two IITs in the field of RF \& Microwave Engineering. His areas of interest include Broadband and Wireless Communication, Telemedicine, e-Governance and Internet of Things. 
